\title{
Article \\ A New Twist: The Combination of Sulbactam/Avibactam Enhances Sulbactam Activity against Carbapenem-Resistant Acinetobacter baumannii (CRAB) Isolates
}

\author{
Fernando Pasteran ${ }^{1}$, Jose Cedano ${ }^{2}$, Michelle Baez ${ }^{2}$, Ezequiel Albornoz ${ }^{1}$, Melina Rapoport ${ }^{1}$, Jose Osteria ${ }^{2}$, \\ Sabrina Montaña ${ }^{3}$, Casin Le ${ }^{2}$, Grace Ra ${ }^{2}$, Robert A. Bonomo ${ }^{4,5,6}$, Marcelo E. Tolmasky ${ }^{2}$ (D) Mark Adams ${ }^{7}$, \\ Alejandra Corso ${ }^{1}$ (D) and Maria Soledad Ramirez ${ }^{2, *(D)}$
}

1 National/Regional Reference Laboratory for Antimicrobial Resistance (NRL), Servicio Antimicrobianos, Instituto Nacional de Enfermedades Infecciosas, ANLIS “Dr. Carlos G. Malbrán”, C1282AFF Buenos Aires, Argentina; fpasteran@gmail.com or fpasteran@anlis.gob.ar (F.P.); Ealbornoz@anlis.gob.ar (E.A.); Rapoport@anlis.gob.ar (M.R.); Acorso@anlis.gob.ar (A.C.)

2 Center for Applied Biotechnology Studies, Department of Biological Science, College of Natural Sciences and Mathematics, California State University Fullerton, Fullerton, CA 92831-3599, USA;

Jcedano@csu.fullerton.edu (J.C.); michelleb777@csu.fullerton.edu (M.B.); josteria@csu.fullerton.edu (J.O.); casle@Fullerton.edu (C.L.); gracera@csu.fullerton.edu (G.R.); mtolmasky@fullerton.edu (M.E.T.)

3 Laboratorio de Bacteriología Clínica, Departamento de Bioquímica Clínica, Hospital de Clínicas José de San Martín, Facultad de Farmacia y Bioquímica, C1120 Buenos Aires, Argentina; sabri.mon@hotmail.com

check for updates

Citation: Pasteran, F.; Cedano, J.; Baez, M.; Albornoz, E.; Rapoport, M.; Osteria, J.; Montaña, S.; Le, C.; Ra, G.; Bonomo, R.A.; et al. A New Twist: The Combination of Sulbactam/ Avibactam Enhances Sulbactam Activity against CarbapenemResistant Acinetobacter baumannii (CRAB) Isolates. Antibiotics 2021, 10, 577. https://doi.org/10.3390/ antibiotics 10050577

Academic Editor: Maria

Lina Mezzatesta

Received: 29 April 2021

Accepted: 11 May 2021

Published: 13 May 2021

Publisher's Note: MDPI stays neutral with regard to jurisdictional claims in published maps and institutional affiliations.

Copyright: (๑) 2021 by the authors. Licensee MDPI, Basel, Switzerland. This article is an open access article distributed under the terms and conditions of the Creative Commons Attribution (CC BY) license (https:// creativecommons.org/licenses/by/ $4.0 /)$.
4 Research Service and GRECC, Louis Stokes Cleveland Department of Veterans Affairs Medical Center, Cleveland, OH 44106, USA; Robert.Bonomo@va.gov

5 Departments of Medicine, Pharmacology, Molecular Biology and Microbiology, Biochemistry, Proteomics and Bioinformatics, Case Western Reserve University School of Medicine, Cleveland, OH 44106, USA

6 CWRU-Cleveland VAMC Center for Antimicrobial Resistance and Epidemiology (Case VA CARES), Cleveland, $\mathrm{OH} 44106$, USA

7 The Jackson Laboratory, Farmington, CT 06032, USA; madams@jcvi.org

* Correspondence: msramirez@Fullerton.edu; Tel.: +1-657-278-4562

Abstract: An increasing number of untreatable infections are recorded every year. Many studies have focused their efforts on developing new $\beta$-lactamase inhibitors to treat multi-drug resistant (MDR) isolates. In the present study, sulbactam/avibactam and sulbactam/relebactam combination were tested against 187 multi-drug resistant (MDR) Acinetobacter clinical isolates; both sulbactam/avibactam and sulbactam/relebactam restored sulbactam activity. A decrease $\geq 2$ dilutions in sulbactam MICs was observed in $89 \%$ of the isolates when tested in combination with avibactam. Sulbactam/relebactam was able to restore sulbactam susceptibility in $40 \%$ of the isolates. In addition, the susceptibility testing using twenty-three A. baumannii AB5075 knockout strains revealed potential sulbactam and/or sulbactam/avibactam target genes. We observed that diazabicyclooctanes (DBOs) $\beta$-lactamase inhibitors combined with sulbactam restore sulbactam susceptibility against carbapenem-resistant Acinetobacter clinical isolates. However, relebactam was not as effective as avibactam when combined with sulbactam. Exploring novel combinations may offer new options to treat Acinetobacter spp. infections, especially for widespread oxacillinases and metallo- $\beta$-lactamases (MBLs) producers.

Keywords: Acinetobacter; carbapenem-resistance; sulbactam; avibactam; relebactam

\section{Introduction}

Infections caused by antibiotic-resistant bacteria are increasing in frequency, resulting in significant patient morbidity and mortality [1,2]. Acinetobacter baumannii is a nosocomial pathogen frequently resistant to multiple drugs. A. baumannii causes pneumonia, bacteremia, and wound infections with associated high mortality rates. The most problematic strains are those resistant to carbapenems (carbapenem-resistant A. baumannii, CRAB). In most cases, resistance is caused by either chromosomal or plasmid-mediated class D oxacillinases (bla $a_{\mathrm{OXA}}$ ) [3-9], but recently, bla $a_{\mathrm{NDM}-1}$ has also been increasingly observed [8,10-15]. 
The advent of CRAB left few active antimicrobials, and unfortunately, these "last resort" antibiotics (polymyxins, tigecycline, minocycline, and amikacin) are limited in efficacy and toxic [16]. Interestingly, sulbactam, a $\beta$-lactamase inhibitor of Ambler class A enzymes, exhibits an inherent antibacterial activity against some bacterial species, including Neisseria gonorrhoeae, Bacteroides fragilis, and Acinetobacter spp. [17]. Although ampicillinsulbactam effectively treated pneumonia, bacteremia, and other nosocomial infections caused by $A$. baumannii, increasing resistance to this combination is becoming ever more common [18-20].

Many recent studies focused on the development of new $\beta$-lactamase inhibitors to treat multi-drug resistant (MDR) infections [21-28]. This research resulted in the design of important types of $\beta$-lactamase inhibitors that include diazabicyclooctanes (DBOs) and boronate-based compounds $[25,29]$. A promising DBO inhibitor is avibactam, a class $A$ and $C \beta$-lactamase inhibitor with some activity against class D enzymes (OXA-48like) $[25,27,30,31]$. While this inhibitor showed promising activity in combination with ceftazidime to treat infections caused by pathogens with extreme resistance, it failed against Acinetobacter [28]. In addition, a new FDA-approved DBO, relebactam, was effective in combination with imipenem against KPC carbapenemases, AmpCs (class C), and/or extended-spectrum $\beta$-lactamases (ESBLs) produced by Enterobacterales [24,26,30].

On the other hand, sulbactam combined with avibactam exhibited a synergistic activity against class D carbapenemase-producing A. baumannii isolates [31]. Previous studies evaluated the activity of sulbactam combined with other antibiotics [32-35]. However, the performance of the combination of sulbactam with avibactam or relebactam against carbapenem-resistant Acinetobacter is unknown. By interacting with multiple penicillin binding proteins and $\beta$-lactamases, we hypothesized that the combination of sulbactam and avibactam or relebactam further sensitizes A. baumannii and reduces MICs.

\section{Results}

Of the 187 selected Acinetobacter strains, including non-ESBL/non-CRAB $(\mathrm{n}=4), \mathrm{ESBL}$ ( $\mathrm{n}=7$ as follows, 5 PER-2, 1 VEB-1, 1 CTX-M-15), OXA-23 $(\mathrm{n}=105)$, OXA-58 $(\mathrm{n}=8)$, OXA-143 $(\mathrm{n}=2)$, ISAba1-bla OXA-66 $(\mathrm{n}=1)$, IMP-1 $(\mathrm{n}=12)$ and NDM-1 $(\mathrm{n}=48)$ producers, $26 / 187(14 \%)$ were inhibited by $\leq 4 \mathrm{mg} / \mathrm{L}$ of sulbactam alone. Only $10 / 116(9 \%)$ of OXA producers and none of the NDM producers were susceptible to sulbactam (MIC $\leq 4 \mathrm{mg} / \mathrm{L}$ ) (Figure 1A). Narrow-spectrum $\beta$-lactamases such as TEM-1, a common contributor to sulbactam resistance in Acinetobacter (19), were detected only in 9 isolates; 8 isolates also produced OXA-23 and one OXA-58. Co-production of ESBL among CRAB, another attributable cause of sulbactam resistance, was observed in 17 isolates: PER-7 and PER-2 ESBL were detected among 14 NDM and 3 OXA-58 producers, respectively. The MICs of sulbactam showed a bi-modal distribution with a first mode of $16 \mathrm{mg} / \mathrm{L}$, corresponding to OXA producers, and a second mode presumable above the highest evaluated value ( $>64 \mathrm{mg} / \mathrm{L}$ ) due to NDM producers (Figure 1A). 

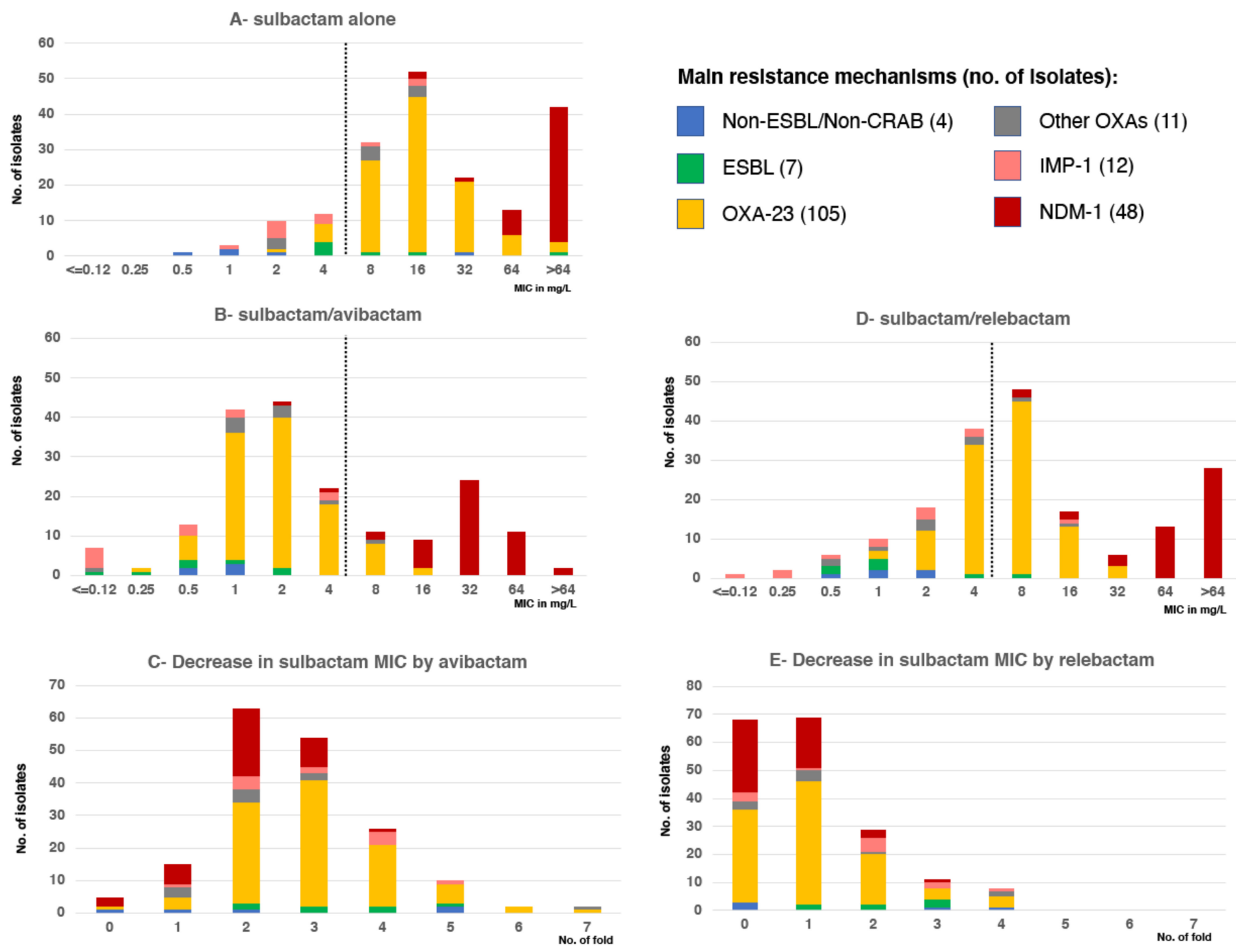

Figure 1. MIC frequency distribution of sulbactam/avibactam, sulbactam/relebactam and sulbactam alone according to the main resistance mechanism. Number of isolates with the indicated sulbactam (A) sulbactam/avibactam (B) or sulbactam/relebactam (D) MIC value, according to the resistant mechanisms. No. isolates with the indicated concentration decrease in sulbactam MIC by addition of avibactam $(\mathbf{C})$ or relebactam (E) according to the resistant mechanisms. The dotted line indicates the breakpoint value that defines susceptible/resistant (A,B,D) according to definitions.

\subsection{Sulbactam/Avibactam Susceptibility Testing of Acinetobacter spp. Clinical Strains}

We observed that 129/187 (69\%) Acinetobacter spp. isolates were inhibited by $4 \mathrm{mg} / \mathrm{L}$ of sulbactam when combined with $4 \mathrm{mg} / \mathrm{L}$ avibactam (Figure 1B). Remarkably, 105/116 (91\%) of OXA producers showed MIC equal to or less than $4 \mathrm{mg} / \mathrm{L}$ for the sulbactam/avibactam combination. NDM producers showed a reduction in the MICs of sulbactam due to the addition of avibactam, although MIC values were above $4 \mathrm{mg} / \mathrm{L}$ for all but one isolate (Figure 1B). The MICs of sulbactam/avibactam also showed a bi-modal distribution but with its modes displaced toward lower MICs. The first mode of $2 \mathrm{mg} / \mathrm{L}$ (3-fold lower when compared to sulbactam alone) corresponded to OXA producers, and the second mode of $32 \mathrm{mg} / \mathrm{L}$ to NDM producers. The $\mathrm{MIC}_{50}$ and $\mathrm{MIC}_{90}$ of sulbactam/avibactam were 2 and $4 \mathrm{mg} / \mathrm{L}$ for OXA producers compared to 16 and $64 \mathrm{mg} / \mathrm{L}$ for sulbactam alone, respectively. Among NDM producers, the $\mathrm{MIC}_{50}$ and $\mathrm{MIC}_{90}$ values of $>64 \mathrm{mg} / \mathrm{L}$ for sulbactam alone decreased to 32 and $64 \mathrm{mg} / \mathrm{L}$ for sulbactam/avibactam, respectively. An experiment using an expanded range of concentrations using as highest value $1024 \mathrm{mg} / \mathrm{L}$ on a subset of NDM producers $(\mathrm{n}=40 / 48)$ showed an $\mathrm{MIC}_{50}$ of $256 \mathrm{mg} / \mathrm{L}$ for sulbactam alone and $32 \mathrm{mg} / \mathrm{L}$ 

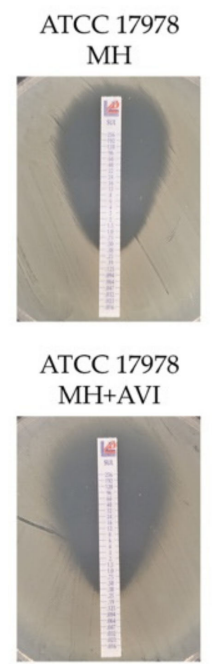

for sulbactam/avibactam. This result indicated that there is an enhancement of sulbactam activity by avibactam in this group of strains.

The average sulbactam MIC decrease (range) by avibactam was: 3-fold decrease (0-5) for non-ESBL/non-CRE, 4-fold decrease (2-9) for ESBL, 3-fold decrease (0-7) for OXA-23, 2.4-fold decrease (2-7) for other OXAs, 2.4-fold decrease (1-5) for IMP-1 and 2-fold decrease (0-4) for NDM producers (Figure 1C).

A total of $159(89 \%)$ of the strains showed an equal or greater than twofold decrease in the sulbactam MIC by avibactam: 32/40 (80\%) NDMs, 11/12 (92\%) IMP-1, 106/116 (91\%) OXAs, $7 / 7$ (100\%) ESBL and 3/5 (60\%) non-ESBL/non-CRAB (Figure 1C). Sulbactam enhanced activity by avibactam was confirmed in selected strains by gradient diffusion method (E-test) with commercial sulbactam strips (Liofilchem) supporting the previous observation (Figure 2).
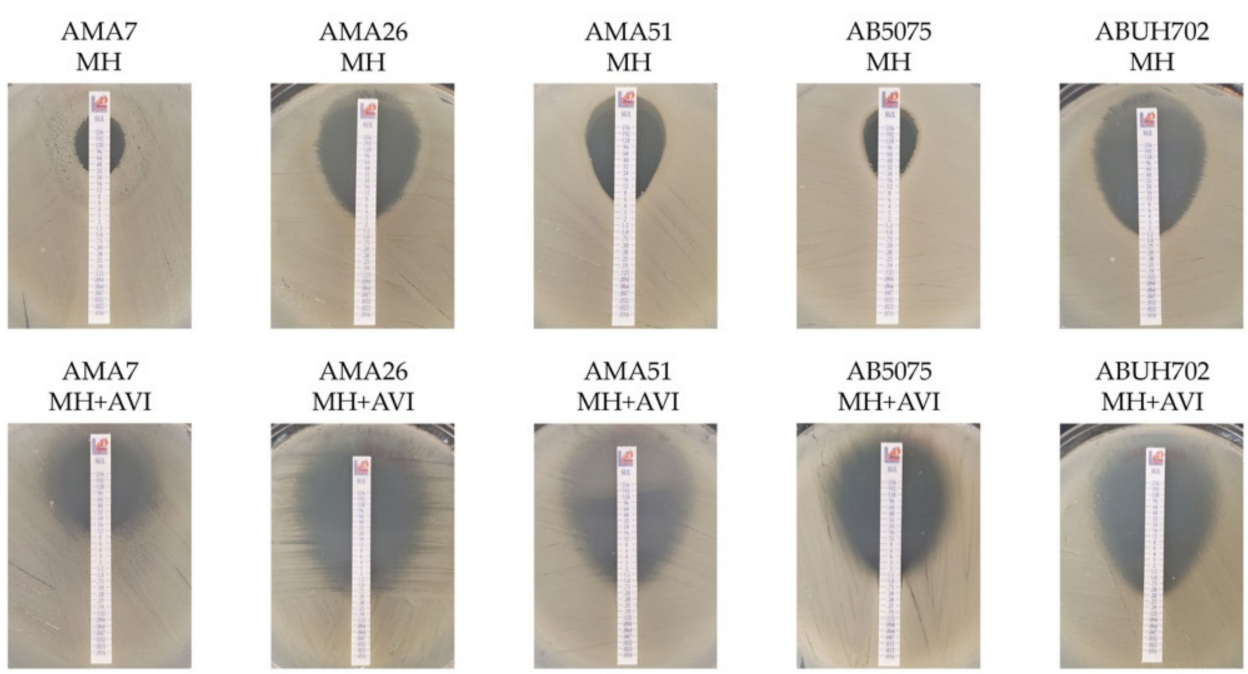

Figure 2. Gradient diffusion (E-test) of sulbactam/avibactam (MH + AVI) and sulbactam (MH) in selected representative strains.

Among OXA-producers, only one A. baumannii OXA-23 (M15094/AMA116) showed identical MIC values for sulbactam alone and sulbactam/avibactam (MIC $16 \mathrm{mg} / \mathrm{L}$ ). Other 8 OXA-producers $(7 \mathrm{~A}$. baumannii and $1 \mathrm{~A}$. sp. genomospecies 10 ) exhibited a 1 dilution decreased in sulbactam MIC by avibactam: 3 isolates already susceptible to sulbactam alone (OXA-58) and 5 resistant isolates (OXA-23).

\subsection{Sulbactam/Relebactam Susceptibility Testing of Acinetobacter spp. Clinical Strains}

With the sulbactam/relebactam combination, 73/187 (39\%) Acinetobacter spp. isolates were inhibited by $4 \mathrm{mg} / \mathrm{L}$ (Figure 1D). The difference in activity with avibactam was due to a smaller proportion of OXA producers $(52 / 116,45 \%)$ inhibited with the combination of sulbactam/relebactam (Figure 1E). All 9 Acinetobacter isolates producing class $\mathrm{D} \beta$-lactamases that were not inhibited by avibactam/sulbactam were also resistant to avibactam/relebactam. The MICs of sulbactam/relebactam also showed a bi-modal distribution with $\mathrm{MIC}_{50}$ and $\mathrm{MIC}_{90}$ of sulbactam/relebactam of 8 and $16 \mathrm{mg} / \mathrm{L}$ for OXA producers compared to 16 and $64 \mathrm{mg} / \mathrm{L}$ for sulbactam alone. Among NDM producers, the $\mathrm{MIC}_{50}$ values were $64 \mathrm{mg} / \mathrm{L}$ for sulbactam/relebactam alone compared to $256 \mathrm{mg} / \mathrm{L}$ for sulbactam.

For strains harboring MBLs, colistin and tigecycline were the only tested comparators with uniform in vitro activity ( $100 \%$ and $94 \%$ susceptible, respectively). Only $18 \%$ of NDM producers were susceptible to amikacin. Conversely, comparators among OXA producers were less active than the sulbactam/avibactam combination: $86 \%$ and $85 \%$ of susceptibility to colistin and tigecycline, respectively. Ceftazidime was only active against non-ESBL/non-CRAB isolates, while ceftazidime/avibactam was not active against CRAB 
and showed a modest $14 \%$ susceptibility against ESBL producers. Imipenem was uniformly active against non-CRAB isolates, but only $2 \%$ of CRABs (all OXA-58 producers) were susceptible to this carbapenem.

\subsection{Sulbactam/Avibactam Susceptibility Testing of A. baumannii Knockout Strains}

The potentiation of sulbactam activity by avibactam was observed in carbapenemases refractory to DBOs inhibition, as OXAs commonly found in Acinetobacter, suggesting a mode of action in these species unrelated to the reported inhibitory capacity. To reveal the potential sulbactam/avibactam targets, twenty-three A. baumannii AB5075 knockout strains were tested to identify potential sulbactam/avibactam targets. Knockout mutant in genes related with efflux pumps, two components system, cell wall synthesis genes, among others, were used. A. baumannii AB5075 MIC to sulbactam was $24 \mathrm{mg} / \mathrm{L}$ and showed a 16 times reduction in sulbactam MIC by addition of avibactam (Table 1 ).

Table 1. Sulbactam MICs against knockout A. baumannii strains using Mueller-Hinton agar with and without avibactam supplementation.

\begin{tabular}{|c|c|c|}
\hline Strains & MH & $\mathrm{MH}+\mathrm{AVI} 4 \mathrm{mg} / \mathrm{L}$ \\
\hline AB5075 & 24 & 1.5 \\
\hline $\mathrm{AB} 5075 \Delta m r c B$ & 3 & 1.5 \\
\hline $\mathrm{AB} 5075 \Delta m r e B$ & 3 & 1.5 \\
\hline AB5075 $\triangle P B P G$ & 8 & $8-12$ \\
\hline $\mathrm{AB} 5075 \Delta b f m R$ & 24 & 12 \\
\hline $\mathrm{AB} 5075 \Delta x e r D$ & 16 & 8 \\
\hline $\mathrm{AB} 5075 \Delta a d v A$ & 16 & 16 \\
\hline $\mathrm{AB} 5075 \Delta a d e B$ & 16 & 16 \\
\hline $\mathrm{AB} 5075 \Delta x e r C$ & 18 & 8 \\
\hline $\mathrm{AB} 5075 \Delta b f m S$ & 16 & 6 \\
\hline AB5075 $\Delta a d e A$ & 24 & 8 \\
\hline $\mathrm{AB} 5075 \Delta l p x B$ & 24 & 8 \\
\hline $\mathrm{AB} 5075 \Delta m r e D$ & 24 & 8 \\
\hline $\mathrm{AB} 5075 \Delta d k_{s} A$ & 24 & 8 \\
\hline $\mathrm{AB} 5075 \Delta r e c A$ & 32 & 12 \\
\hline AB5075 $\Delta a d e K$ & 24 & 6 \\
\hline $\mathrm{AB} 5075 \triangle a m p \mathrm{C}$ & 24 & 6 \\
\hline AB5075 $\triangle$ OXA-69 & 16 & 4 \\
\hline $\mathrm{AB} 5075 \Delta m r e \mathrm{C}$ & 16 & 4 \\
\hline $\mathrm{AB} 5075 \Delta m r d B$ & 16 & 4 \\
\hline $\mathrm{AB} 5075 \Delta m l t B$ & 16 & $1.5-3$ \\
\hline $\mathrm{AB} 5075 \Delta E l s L$ & 24 & 4 \\
\hline $\mathrm{AB} 5075 \Delta d n a K$ & 24 & 4 \\
\hline $\mathrm{AB} 5075 \Delta a d e R$ & 32 & 3 \\
\hline
\end{tabular}

The results observed with A. baumannii AB5075 mutants can be classified into three large groups.

i. Mutations affecting the activity of sulbactam alone: when comparing sulbactam MIC in the wild-type strains and isogenic mutants, a decrease in MIC values from 24 to $3 \mathrm{mg} / \mathrm{L}$ was observed in the $A B 5075 \Delta m r c B$ and $A B 5075 \Delta m r e B$, suggesting a potential role in sulbactam susceptibility (interaction). The $m r c B$ and $m r e B$ genes, which code for a transglycosylase-transpeptidase and a cytoskeletal protein, respectively, were previously reported as genes involved in the formation of the peptidoglycan synthesis. Potentiation of sulbactam activity by avibactam is also lost in these mutant strains. Additionally, the A. baumannii AB5075 $\triangle$ PBPG MIC of sulbactam is 1.5-fold lower than that measured for A. baumannii AB5075. PBPs are known to be sulbactam targets [36]. Previous reports demonstrated that the antibacterial activity of sulbactam was mediated through inhibition of PBP1 and PBP3. 
ii. Mutations that result in loss of avibactam enhancement of sulbactam activity: a 1 or 0 -fold decrease in the MIC values comparing sulbactam with sulbactam/avibactam MICs was observed in seven A. baumannii knockout strains (AB5075 $\Delta b \mathrm{fmR}, \mathrm{AB} 5075 \Delta P$ $B P G, \mathrm{AB} 5075 \Delta a d v A, \mathrm{AB} 5075 \Delta a d e B, \mathrm{AB} 5075 \Delta m r c B, \mathrm{AB} 5075 \Delta x e r D$, and $\mathrm{AB} 5075 \Delta m r e B)$. These results identified potential sulbactam and/or sulbactam/avibactam targets (Table 1).

iii. Mutations that do not affect susceptibility to sulbactam or potentiation of sulbactam by avibactam: $\leq 2$-fold decrease in the MIC was observed in twelve A. baumannii AB5075 knockout strains comparing sulbactam with sulbactam/avibactam MIC. We can rule out that these mutated genes are involved in the mechanisms of action (or interaction) of sulbactam and avibactam.

\section{Discussion}

Infections with the hospital-acquired bacterium Acinetobacter are extremely difficult to treat. While the development of new antibiotics remains one option in the fight against strains resistant to those currently available, an attractive (and faster) alternative is the development of adjuvant therapies to restore the efficacy of existing antibiotics. A preliminary in vitro study has shown promising results with avibactam adjuvant approaches combined with sulbactam [31]. In this work we contributed new knowledge to support sulbactam/avibactam interaction.

We observed that the addition of DBOs restores sulbactam susceptibility. This interaction between $\mathrm{DBO}$ and sulbactam presented the following characteristics: (i) was more potent in serine carbapenemase-producing strains, such as oxacillinases, but it was also manifested to a lesser extent in NDM-producing strains; (ii) was not related to the DBO concentration as a dose-response relationship was not observed in MICs when both DBOs were tested using twice the recommended concentration ( $8 \mathrm{mg} / \mathrm{L}$ ) (not shown); (iv) was not related to the $\beta$-lactamase inhibitory capacity described for DBOs; and (v) avibactam was more potent in restoring sulbactam susceptibility than relebactam. The differences observed between avibactam and relebactam would support these hypotheses, the larger molecular size of the second DBO (molar mass $265.24 \mathrm{~g} / \mathrm{mol} \mathrm{g} \cdot \mathrm{mol}^{-1} \mathrm{vs} .348 .37 \mathrm{~g} \cdot \mathrm{mol}^{-1}$, respectively) could be responsible for a reduced capability to penetrate the outer membrane and reach its targets.

Recently, ETX2514, named durlobactam, a new DBO inhibitor derived from avibactam (resulting from the addition of a double bond between C3 and C4 and a methyl group in C3 position compared to avibactam), significantly increased the susceptibility of Acinetobacter clinical isolates when it was combined with sulbactam [22]. Sulbactam/durlobactam has an $\mathrm{MIC}_{50} / \mathrm{MIC}_{90}$ of 1 and $2 \mathrm{mg} / \mathrm{L}$ against a global collection of strains. These values are comparable to those obtained in this work for the sulbactam/avibactam combination for OXA-23 producers ( 2 and $4 \mathrm{mg} / \mathrm{L}$, respectively) [22]. However, the combination with durlobactam was not effective for NDM strains with $\mathrm{MIC}_{50 / 90}$ comparable to those of sulbactam alone, unlike avibactam, which showed a slight improvement in sulbactam MICs in these strains. In this context, the potent synergy observed between sulbactam and avibactam indicates that there could be different targets for each DBO.

We were unable to detect a known resistant mechanism compromising sulbactam activity. Only a small proportion of the strains presented narrow-spectrum or extended spectrum $\beta$-lactamases that affect sulbactam. No mutations in the genes coding for PBPs were observed in a previously analyzed subset of NDM producers [15]. Taken together, these results suggest that sulbactam/avibactam synergy could have an effect on other targets in MDR strains.

We explored the possible contribution in resistance to sulbactam and its interaction with avibactam of genes related with efflux pumps, two components system, cell wall synthesis genes. We observed that $m r c B$ and $m r e B$ were two key components, in addition to the coding genes for PBPs, for phenotypic resistance to sulbactam. $m r c B$, which codes for a transglycosylase-transpeptidase, and $m r e B$, which codes for cytoskeletal proteins, could 
play a key role in maintaining vital (or alternate) processes in wall synthesis in the presence of sulbactam, which can explain in part the observed sulbactam MIC reduction when they are inactivated. Potentiation of sulbactam activity by avibactam is also lost in these mutant strains, suggesting that conserved $m r c B$ and $m r e B$ are a necessary (but not sufficient) prerequisite for avibactam interactions. More studies to discern the possible interplay of sulbactam and sulbactam/avibactam on $m r e B$ and $m r c B$ are needed. We also identified key genes that seem necessary to guarantee the interaction between sulbactam and avibactam. BfmRS, a global regulatory system in Acinetobacter, is a key challenge for antibiotic therapy. BfmRS per se has been shown to increase the ability of Acinetobacter to grow in the presence of diverse antibiotics and tolerate transient, high-level antibiotic exposures [37]. As we observed, avibactam (with sulbactam) may interfere or bypass the normal protection from $\beta$-lactam toxicity mediated by BfmRS. advA (antibiotic susceptibility and division protein of Acinetobacter) is another essential gene for A. baumannii growth. It has been shown that reduced AdvA levels modulated antibiotic susceptibility and generate a selective pattern of hypersensitivity to fluoroquinolones and $\beta$-lactams [38]. Avibactam could be a hypothetical mediator for sulbactam hypersensitivity through $a d v A$ interaction. Identification of other genes potentially involved in the interaction between sulbactam and avibactam, such as XerCD, tyrosine recombinases that catalyze the resolution of dimeric chromosomes [39], opens up new possibilities in target identification for designing new drug combinations.

In summary, sulbactam/DBOs, especially in combination with avibactam, demonstrated potent antibacterial activity against recent, geographically diverse clinical isolates of Acinetobacter, including XDR isolates such as widespread oxacillinase producers. The addition of a DBO to sulbactam appears to be a very promising strategy for managing difficult-to-treat Acinetobacter infections. We identified at least 6 genes that could participate in the interaction of sulbactam with avibactam. Understanding this interaction will clarify how $A$. baumannii optimizes its ability to grow under antibiotics exposition. Further extensive in vitro and in vivo studies have to be performed to confirm the potential use of these adjuvants as therapeutic alternatives.

\section{Materials and Methods}

\subsection{Bacterial Strains}

A representative panel of 187 ESBL-producers and CR Acinetobacter spp. clinical strains were used to test sulbactam or sulbactam in combination with avibactam or relebactam (Table S1). Among the included strains 152 were $A$. baumannii, including previously well-characterized strains such as AB5075, AB0057 and ABUH702 [40-42], and 35 corresponded to non-baumannii species. Among these isolates, 4 were non-ESBL/non-CRAB, 7 were ESBL producing, while 176 were carbapenem-resistant strains. The ATCC 17,978 A. baumannii, ATCC 700,903 Klebsiella pneumoniae, ATCC 25,922 Escherichia coli, and ATCC 35,218 E. coli strains were used as control. In addition, 23 mutant strains from Manoil Lab (Washington, DC, USA) were used to test sulbactam/avibactam activity. Among them we selected AB5075 $\Delta a d e R, \mathrm{AB} 5075 \Delta b \mathrm{fmR}, \mathrm{AB} 5075 \Delta b \mathrm{fmS}, \mathrm{AB} 5075 \Delta P B P G, \mathrm{AB} 5075 \Delta a d v A$, $\mathrm{AB} 5075 \Delta a d e B, \mathrm{AB} 5075 \Delta a m p C, \mathrm{AB} 5075 \Delta \mathrm{OXA}-69, \mathrm{AB} 5075 \Delta a d e A, \mathrm{AB} 5075 \Delta m r d B, \mathrm{AB} 5075 \Delta m l t B$, $\mathrm{AB} 5075 \Delta m r c B, \mathrm{AB} 5075 \Delta a d e K, \mathrm{AB} 5075 \Delta r e c A, \mathrm{AB} 5075 \Delta x e r D, \mathrm{AB} 5075 \Delta x e r C, \mathrm{AB} 5075 \Delta l p x B$, $\mathrm{AB} 5075 \Delta m r e B, \mathrm{AB} 5075 \Delta m r e C, \mathrm{AB} 5075 \Delta m r e D, \mathrm{AB} 5075 \Delta E l s L, \mathrm{AB} 5075 \Delta d n a K, \mathrm{AB} 5075 \Delta d k s A$.

\subsection{Antimicrobial Susceptibility Assay}

MICs against sulbactam (range $0.12-512 \mathrm{mg} / \mathrm{L}$ ), sulbactam plus $4 \mathrm{mg} / \mathrm{L}$ avibactam (range 0.12-64 mg/L) and sulbactam plus $4 \mathrm{mg} / \mathrm{L}$ relebactam (range $0.12-64 \mathrm{mg} / \mathrm{L}$ ) were performed using the reference methods agar dilution according to CLSI Standards [43]. Because breakpoints are available for sulbactam alone, $4 \mathrm{mg} / \mathrm{L}$ was applied for this analysis based on the CLSI susceptible breakpoint of $8 / 4 \mathrm{mg} / \mathrm{L}$ for ampicillin/sulbactam for Acinetobacter spp. [44,45]. The in vitro activity of other comparators, such as imipenem, colistin, tigecycline, amikacin, ceftazidime (alone and in combination with $4 \mathrm{mg} / \mathrm{L}$ of avibactam) were also evaluated by dilution methods at one facility. 
Supplementary Materials: The following are available online at https:/ / www.mdpi.com/article/10 .3390 /antibiotics10050577/s1, Table S1: Features of Acinetobacter isolates.

Author Contributions: Conceptualization, F.P., R.A.B. and M.S.R.; methodology, F.P., J.C., M.A., and M.S.R.; formal analysis, F.P., J.C., C.L., G.R., R.A.B. and M.S.R.; investigation, F.P., J.C., M.B., E.A., M.R., J.O., C.L., G.R., R.A.B. and M.S.R.; resources, F.P., M.A., A.C. and M.S.R.; data curation, F.P., J.C., M.B., E.A., M.R., J.O., C.L., G.R. and M.S.R.; writing-original draft preparation, F.P., J.C., S.M., R.A.B., M.E.T., M.A. and M.S.R.; writing-review and editing, F.P., J.C., S.M., R.A.B., M.E.T., M.A. and M.S.R.; supervision, F.P., R.A.B. and M.S.R.; funding acquisition, R.A.B. and M.S.R. All authors have read and agreed to the published version of the manuscript.

Funding: The authors' work cited in this review article was funded by Public Health Service Grants SC3GM125556 (M.S.R.), R01AI100560 (R.A.B.), R01AI063517 (to R.A.B.), R01AI072219 (to R.A.B.), and 2R15 AI047115(to M.E.T.) from the National Institutes of Health and VA 1I01BX001974 (to R.A.B.) from the Cleveland Department of Veterans Affairs. The content is solely the responsibility of the authors and does not necessarily represent the official views of the National Institutes of Health or the Department of Veterans Affairs. Also, ANLIS: Regular federal budget from Ministry of Health, Argentina.

Institutional Review Board Statement: Not applicable.

Informed Consent Statement: Not applicable.

Data Availability Statement: Not applicable.

Acknowledgments: We are indebted to those Institutions and professionals of the antimicrobial surveillance systems who referred the clinical isolates to the National Reference Laboratory for the repository collection of the NRL. We want to thank the INVERA-Foundation "investigacion en Resistencia a los Antimicrobianos-Argentina" and Laboratorios Britania for providing relebactam and avibactam, respectively.

Conflicts of Interest: The authors declare no conflict of interest.

\section{References}

1. Boucher, H.W.; Talbot, G.H.; Bradley, J.S.; Edwards, J.E.; Gilbert, D.; Rice, L.B.; Scheld, M.; Spellberg, B.; Bartlett, J. Bad bugs, no drugs: No ESKAPE! An update from the Infectious Diseases Society of America. Clin. Infect. Dis. 2009, 48, 1-12. [CrossRef] [PubMed]

2. Rice, L.B. Federal funding for the study of antimicrobial resistance in nosocomial pathogens: No ESKAPE. J. Infect. Dis 2008, 197, 1079-1081. [CrossRef] [PubMed]

3. Evans, B.A.; Brown, S.; Hamouda, A.; Findlay, J.; Amyes, S.G. Eleven novel OXA-51-like enzymes from clinical isolates of Acinetobacter baumannii. Clin. Microbiol. Infect. 2007, 13, 1137-1138. [CrossRef] [PubMed]

4. Evans, B.A.; Amyes, S.G. OXA beta-lactamases. Clin. Microbiol. Rev. 2014, 27, 241-263. [CrossRef]

5. Bush, K.; Bradford, P.A. Epidemiology of beta-lactamase-producing pathogens. Clin. Microbiol. Rev. 2020, 33. [CrossRef]

6. Heritier, C.; Dubouix, A.; Poirel, L.; Marty, N.; Nordmann, P. A nosocomial outbreak of Acinetobacter baumannii isolates expressing the carbapenem-hydrolysing oxacillinase OXA-58. J. Antimicrob. Chemother. 2005, 55, 115-118. [CrossRef]

7. Jeannot, K.; Diancourt, L.; Vaux, S.; Thouverez, M.; Ribeiro, A.; Coignard, B.; Courvalin, P.; Brisse, S. Molecular epidemiology of carbapenem non-susceptible Acinetobacter baumannii in France. PLoS ONE 2014, 9, e115452. [CrossRef]

8. Rodriguez, C.H.; Nastro, M.; Famiglietti, A. Carbapenemases in Acinetobacter baumannii. Review of their dissemination in Latin America. Rev. Argent. Microbiol. 2018, 50, 327-333. [CrossRef]

9. Ramirez, M.S.; Bonomo, R.A.; Tolmasky, M.E. Carbapenemases: Transforming Acinetobacter baumannii into a Yet More Dangerous Menace. Biomolecules 2020, 10, 720. [CrossRef]

10. Yang, J.; Chen, Y.; Jia, X.; Luo, Y.; Song, Q.; Zhao, W.; Wang, Y.; Liu, H.; Zheng, D.; Xia, Y.; et al. Dissemination and characterization of NDM-1-producing Acinetobacter pittii in an intensive care unit in China. Clin. Microbiol. Infect. 2012, 18, E506-E513. [CrossRef]

11. Tran, D.N.; Tran, H.H.; Matsui, M.; Suzuki, M.; Suzuki, S.; Shibayama, K.; Pham, T.D.; Van Phuong, T.T.; Dang, D.A.; Trinh, H.S.; et al. Emergence of New Delhi metallo-beta-lactamase 1 and other carbapenemase-producing Acinetobacter calcoaceticus-baumannii complex among patients in hospitals in Ha Noi, Viet Nam. Eur. J. Clin. Microbiol. Infect. Dis. 2017, 36, 219-225. [CrossRef]

12. Pillonetto, M.; Arend, L.; Vespero, E.C.; Pelisson, M.; Chagas, T.P.; Carvalho-Assef, A.P.; Asensi, M.D. First report of NDM-1producing Acinetobacter baumannii sequence type 25 in Brazil. Antimicrob. Agents Chemother. 2014, 58, 7592-7594. [CrossRef]

13. El-Mahdy, T.S.; Al-Agamy, M.H.; Al-Qahtani, A.A.; Shibl, A.M. Detection of blaOXA-23-like and blaNDM-1 in Acinetobacter baumannii from the Eastern Region, Saudi Arabia. Microb. Drug Resist. 2017, 23, 115-121. [CrossRef]

14. Beigverdi, R.; Sattari-Maraji, A.; Emaneini, M.; Jabalameli, F. Status of carbapenem-resistant Acinetobacter baumannii harboring carbapenemase: First systematic review and meta-analysis from Iran. Infect. Genet. Evol. 2019, 73, 433-443. [CrossRef] 
15. Adams, M.D.; Pasteran, F.; Traglia, G.M.; Martinez, J.; Huang, F.; Liu, C.; Fernandez, J.S.; Lopez, C.; Gonzalez, L.J.; Albornoz, E.; et al. Distinct mechanisms of dissemination of NDM-1 metallo- beta-lactamase in Acinetobacter spp. in Argentina. Antimicrob. Agents Chemother. 2020, 64. [CrossRef]

16. Butler, D.A.; Biagi, M.; Tan, X.; Qasmieh, S.; Bulman, Z.P.; Wenzler, E. Multidrug Resistant Acinetobacter baumannii: Resistance by Any Other Name Would Still be Hard to Treat. Curr Infect. Dis. Rep. 2019, 21, 46. [CrossRef]

17. Noguchi, J.K.; Gill, M.A. Sulbactam: A beta-lactamase inhibitor. Clin. Pharm. 1988, 7, 37-51.

18. Yang, Y.; Xu, Q.; Li, T.; Fu, Y.; Shi, Y.; Lan, P.; Zhao, D.; Chen, Q.; Zhou, Z.; Jiang, Y.; et al. OXA-23 Is a Prevalent Mechanism Contributing to Sulbactam Resistance in Diverse Acinetobacter baumannii Clinical Strains. Antimicrob. Agents Chemother. 2019, 63. [CrossRef]

19. Krizova, L.; Poirel, L.; Nordmann, P.; Nemec, A. TEM-1 beta-lactamase as a source of resistance to sulbactam in clinical strains of Acinetobacter baumannii. J. Antimicrob. Chemother. 2013, 68, 2786-2791. [CrossRef]

20. Chiu, C.H.; Lee, H.Y.; Tseng, L.Y.; Chen, C.L.; Chia, J.H.; Su, L.H.; Liu, S.Y. Mechanisms of resistance to ciprofloxacin, ampicillin/sulbactam and imipenem in Acinetobacter baumannii clinical isolates in Taiwan. Int. J. Antimicrob. Agents 2010, 35, 382-386. [CrossRef]

21. Paterson, D.L.; Isler, B.; Stewart, A. New treatment options for multiresistant gram negatives. Curr. Opin. Infect. Dis. 2020, 33, 214-223. [CrossRef]

22. McLeod, S.M.; Moussa, S.H.; Hackel, M.A.; Miller, A.A. In Vitro Activity of Sulbactam-Durlobactam against Acinetobacter baumannii-calcoaceticus Complex Isolates Collected Globally in 2016 and 2017. Antimicrob. Agents Chemother. 2020, 64. [CrossRef]

23. Lomovskaya, O.; Nelson, K.; Rubio-Aparicio, D.; Tsivkovski, R.; Sun, D.; Dudley, M.N. The Impact of Intrinsic Resistance Mechanisms on Potency of QPX7728, a New Ultra-Broad-Spectrum Beta-lactamase Inhibitor of Serine and Metallo Beta-Lactamases in Enterobacteriaceae, Pseudomonas aeruginosa, and Acinetobacter baumannii. Antimicrob. Agents Chemother. 2020. [CrossRef]

24. Karaiskos, I.; Galani, I.; Souli, M.; Giamarellou, H. Novel beta-lactam-beta-lactamase inhibitor combinations: Expectations for the treatment of carbapenem-resistant Gram-negative pathogens. Expert Opin. Drug Metab. Toxicol. 2019, 15, 133-149. [CrossRef]

25. Barnes, M.D.; Kumar, V.; Bethel, C.R.; Moussa, S.H.; O’Donnell, J.; Rutter, J.D.; Good, C.E.; Hujer, K.M.; Hujer, A.M.; Marshall, S.H.; et al. Targeting Multidrug-Resistant Acinetobacter spp.: Sulbactam and the Diazabicyclooctenone beta-Lactamase Inhibitor ETX2514 as a Novel Therapeutic Agent. mBio 2019, 10. [CrossRef]

26. Zhanel, G.G.; Lawrence, C.K.; Adam, H.; Schweizer, F.; Zelenitsky, S.; Zhanel, M.; Lagace-Wiens, P.R.S.; Walkty, A.; Denisuik, A.; Golden, A.; et al. Imipenem-Relebactam and Meropenem-Vaborbactam: Two Novel Carbapenem-beta-Lactamase Inhibitor Combinations. Drugs 2018, 78, 65-98. [CrossRef]

27. Wright, H.; Bonomo, R.A.; Paterson, D.L. New agents for the treatment of infections with Gram-negative bacteria: Restoring the miracle or false dawn? Clin. Microbiol. Infect. 2017, 23, 704-712. [CrossRef]

28. Durand-Reville, T.F.; Guler, S.; Comita-Prevoir, J.; Chen, B.; Bifulco, N.; Huynh, H.; Lahiri, S.; Shapiro, A.B.; McLeod, S.M.; Carter, N.M.; et al. ETX2514 is a broad-spectrum beta-lactamase inhibitor for the treatment of drug-resistant Gram-negative bacteria including Acinetobacter baumannii. Nat. Microbiol. 2017, 2, 17104. [CrossRef]

29. Tooke, C.L.; Hinchliffe, P.; Bragginton, E.C.; Colenso, C.K.; Hirvonen, V.H.A.; Takebayashi, Y.; Spencer, J. beta-Lactamases and beta-Lactamase Inhibitors in the 21st Century. J. Mol. Biol. 2019, 431, 3472-3500. [CrossRef]

30. Tooke, C.L.; Hinchliffe, P.; Lang, P.A.; Mulholland, A.J.; Brem, J.; Schofield, C.J.; Spencer, J. Molecular Basis of Class A betaLactamase Inhibition by Relebactam. Antimicrob. Agents Chemother. 2019, 63. [CrossRef]

31. Rodriguez, C.H.; Brune, A.; Nastro, M.; Vay, C.; Famiglietti, A. In vitro synergistic activity of the sulbactam/avibactam combination against extensively drug-resistant Acinetobacter baumannii. J Med. Microbiol. 2020. [CrossRef] [PubMed]

32. Santimaleeworagun, W.; Wongpoowarak, P.; Chayakul, P.; Pattharachayakul, S.; Tansakul, P.; Garey, K.W. In vitro activity of colistin or sulbactam in combination with fosfomycin or imipenem against clinical isolates of carbapenem-resistant Acinetobacter baumannii producing OXA-23 carbapenemases. Southeast Asian J. Trop. Med. Public Health 2011, 42, 890-900. [PubMed]

33. Pei, G.; Mao, Y.; Sun, Y. In vitro activity of minocycline alone and in combination with cefoperazone-sulbactam against carbapenem-resistant Acinetobacter baumannii. Microb. Drug Resist 2012, 18, 574-577. [CrossRef] [PubMed]

34. Poulikakos, P.; Tansarli, G.S.; Falagas, M.E. Combination antibiotic treatment versus monotherapy for multidrug-resistant, extensively drug-resistant, and pandrug-resistant Acinetobacter infections: A systematic review. Eur. J. Clin. Microbiol. Infect. Dis. 2014, 33, 1675-1685. [CrossRef]

35. Kalin, G.; Alp, E.; Akin, A.; Coskun, R.; Doganay, M. Comparison of colistin and colistin/sulbactam for the treatment of multidrug resistant Acinetobacter baumannii ventilator-associated pneumonia. Infection 2014, 42, 37-42. [CrossRef]

36. Penwell, W.F.; Shapiro, A.B.; Giacobbe, R.A.; Gu, R.F.; Gao, N.; Thresher, J.; McLaughlin, R.E.; Huband, M.D.; DeJonge, B.L.; Ehmann, D.E.; et al. Molecular mechanisms of sulbactam antibacterial activity and resistance determinants in Acinetobacter baumannii. Antimicrob. Agents Chemother. 2015, 59, 1680-1689. [CrossRef]

37. Geisinger, E.; Mortman, N.J.; Vargas-Cuebas, G.; Tai, A.K.; Isberg, R.R. A global regulatory system links virulence and antibiotic resistance to envelope homeostasis in Acinetobacter baumannii. PLoS Pathog. 2018, 14, e1007030. [CrossRef]

38. Geisinger, E.; Mortman, N.J.; Dai, Y.; Cokol, M.; Syal, S.; Farinha, A.; Fisher, D.G.; Tang, A.Y.; Lazinski, D.W.; Wood, S.; et al. Antibiotic susceptibility signatures identify potential antimicrobial targets in the Acinetobacter baumannii cell envelope. Nat. Commun. 2020, 11, 4522. [CrossRef] 
39. Lin, D.L.; Traglia, G.M.; Baker, R.; Sherratt, D.J.; Ramirez, M.S.; Tolmasky, M.E. Functional Analysis of the Acinetobacter baumannii XerC and XerD Site-Specific Recombinases: Potential Role in Dissemination of Resistance Genes. Antibiotics 2020, 9, 405. [CrossRef]

40. Jacobs, A.C.; Thompson, M.G.; Black, C.C.; Kessler, J.L.; Clark, L.P.; McQueary, C.N.; Gancz, H.Y.; Corey, B.W.; Moon, J.K.; Si, Y.; et al. AB5075, a Highly Virulent Isolate of Acinetobacter baumannii, as a Model Strain for the Evaluation of Pathogenesis and Antimicrobial Treatments. MBio 2014, 5, e01014-e01076. [CrossRef]

41. Adams, M.D.; Nickel, G.C.; Bajaksouzian, S.; Lavender, H.; Murthy, A.R.; Jacobs, M.R.; Bonomo, R.A. Resistance to colistin in Acinetobacter baumannii associated with mutations in the PmrAB two-component system. Antimicrob. Agents Chemother. 2009, 53, 3628-3634. [CrossRef]

42. Adams, M.D.; Wright, M.S.; Karichu, J.K.; Venepally, P.; Fouts, D.E.; Chan, A.P.; Richter, S.S.; Jacobs, M.R.; Bonomo, R.A. Rapid Replacement of Acinetobacter baumannii Strains Accompanied by Changes in Lipooligosaccharide Loci and Resistance Gene Repertoire. mBio 2019, 10. [CrossRef]

43. Clinical and Laboratory Standards Institute. Methods for Dilution Antimicrobial Susceptibility Tests for Bacteria That Growth Aerobically M7-A10, Ed. M7-A10; Clinical and Laboratory Standards Institute: Wayne, PA, USA, 2018.

44. Clinical and Laboratory Standards Institute. Performance Standards for Antimicrobial Susceptibility Testing; M.100, twenty-Nine Informational Supplement; Clinical and Laboratory Standards Institute: Wayne, PA, USA, 2020.

45. Yang, Q.; Xu, Y.; Jia, P.; Zhu, Y.; Zhang, J.; Zhang, G.; Deng, J.; Hackel, M.; Bradford, P.A.; Reinhart, H. In vitro activity of sulbactam/durlobactam against clinical isolates of Acinetobacter baumannii collected in China. J. Antimicrob. Chemother. 2020, 75, 1833-1839. [CrossRef] 